\title{
Design and implementation of international agricultural and biological engineering expert management system based on WEB mode
}

\author{
Jianbo Shen ${ }^{1}$, Liwei $\operatorname{Tan}^{1}$, Yingkuan Wang ${ }^{1,2^{*}}$ \\ (1. Academy of Agricultural Planning and Engineering, Ministry of Agriculture and Rural Affairs, Beijing 100125, China; \\ 2. Chinese Society of Agricultural Engineering, Beijing 100125, China)
}

\begin{abstract}
Agriculture and biological engineering are the foundation of agricultural modernization, and related countries have also issued relevant policies to guide the development of agriculture and biotechnology. Agricultural and biological engineering experts are the intellectual resources in the field. At present, there is less research on the management and maintenance of agricultural and biological engineering experts, and there is a lack of software systems in this area. In order to realize the management and maintenance of agricultural and biological engineering expert information, a service platform for the international agricultural and biological engineering expert system based on the B/S framework has been developed. The background of the system used C\# as the development language, and the foreground used JavaScript technology and Bootstrap. The software adopted ASP.NET MVC as the web development framework and used the Entity Framework to operate the SQL Server background database. The system has the function of searching and querying agricultural and biological engineering expert information according to keywords, and implements the functions of adding, deleting, and modifying data records, and the function of generating spreadsheets and importing spreadsheet data. The development of this system provides effective management tools for the maintenance and construction of agricultural and biological engineering expert databases and lays a good foundation for the construction of agricultural and biological engineering think tanks.
\end{abstract}

Keywords: agricultural and bioengineering engineering, expert management, WEB mode, conditional search, think tanks, Asp.net MVC, bootstrap, entity framework technology

DOI: $10.25165 /$ j.jjabe.20201306.5759

Citation: Shen J B, Tan L W, Wang Y K. Design and implementation of international agricultural and biological engineering expert management system based on WEB mode. Int J Agric \& Biol Eng, 2020; 13(6): 195-200.

\section{Introduction}

Agriculture and bioengineering are the foundation of modern agriculture. Agricultural biotechnology has been regarded as the core competitiveness of major agricultural countries, and related countries have also issued relevant policies to guide the development of agriculture and biotechnology ${ }^{[1-4]}$. Talent resources in agricultural engineering and biotechnology are the core driving force for innovation in this field. Talent think tanks and talent big data have become hot issues at the moment ${ }^{[4-6]}$. How to collect, mine, analyze, process, and deep dig agricultural and bioengineering expert information is urgently needed. There is little research on information collection, processing and management of international agricultural and biological engineering experts, and a lack of software systems in this respect.

The information on the agricultural and biological engineering expert database is constantly updated, so it needs to be maintained on a regular and dynamic basis. That is, the relevant information of agricultural bioengineering experts needs to be added, deleted or modified in time. Establishing international agricultural and biological engineering expert data service platform based web mode to achieve the maintenance and management of international agricultural and biological engineering expert information can lay a

Received date: 2020-02-27 Accepted date: 2020-08-30

Biographies: Jianbo Shen, $\mathrm{PhD}$, Engineer, research interest: agricultural informatization, Email:lyshenjianbo@163.com; Liwei Tan, Senior Engineer, research interest: agricultural informatization, Email: 155113261@qq.com.

*Corresponding author: Yingkuan Wang, $\mathrm{PhD}$, Researcher, research interest: agricultural mechanization, automatization and informatization. Academy of Agricultural Planning and Engineering, MARA, Beijing 100125, China. Tel: +86-10-59197086, Email: wangyingkuan@163.com. good foundation for the establishment of agricultural and biological engineering expert think tanks.

In order to accelerate the development of agricultural and biological engineering disciplines, it is necessary to establish an agricultural and biological engineering expert information database. The research and construction of an agricultural and biological engineering expert information database service management platform not only plays an important role in promoting the efficiency of expert consultation in agricultural and biological engineering and the improvement of scientific and technological services but also in promoting the academic exchanges of agricultural and biological engineering experts and the rapid development of the discipline are of positive significance.

\section{Demand analysis}

To address the problems of insufficient supply of existing scientific and technological personnel and technological knowledge, poor connection between technological supply and demand and production demand, and poorly targeted scientific and technological information services, the platform categorizes the expertise of the experts, covering all aspects of the agricultural and biological engineering areas, for example, planting, breeding, agricultural materials, agricultural machinery and equipment, etc. And it can provide farmers and agricultural experts with seamless technical exchanges, two-way interaction, and zero-distance contact service connection to lay a good foundation, break the constraints of time and space, and provide personalized customized service docking for more farmers and professional cooperatives. In order to realize the "one-to-one, one-to-many, many-to-many" expert information docking and service platform, the resources of think tanks in the agricultural and biological fields were made full 
use to solve the problems in production, and thus improve the efficiency of the agricultural and biological fields.

At present, more and more countries are paying more attention to agricultural and bioengineering technology, but how to make the best use of agricultural and bioengineering expert resources to serve agricultural and bioengineering fields. The purpose of the establishment of the international agricultural and biological engineering expert database platform is to timely grasp the relevant information of experts in the agricultural and biological engineering fields of various countries, including professional expertise and academic progress, which can get agricultural and bioengineering filed better. At present, because agricultural and biological engineering experts come from different countries, the expert information is electronic files in different formats, and the cost of manual maintenance and management is relatively high. The maintenance and management of agricultural and biological engineering expert information are time-consuming and laborious. According to the actual needs in the field of agricultural and biological engineering, a simple and friendly interface was developed, which based on the $\mathrm{B} / \mathrm{S}$ mode of international agricultural and biological engineering expert maintenance and management platform. B/S mode does not need the client to download any software as long as the client installs a browser, and can implement multipoint to multipoint multimedia communication ${ }^{[7-9]}$. A platform that can improve the efficiency of data maintenance and management of current international agricultural and biological engineering experts was developed. It played an important role in improving the efficiency of management and maintenance. The platform is divided into three modules: data maintenance, data query, and authority management. In the data maintenance module, the functions of adding, deleting, modifying and importing spreadsheets by experts are realized. In the data query module, the search function according to the fields of the expert's name, title, and specialty is realized. In the authority management module, the authority control for different types of users is realized through role management. Using the developed software platform to maintain and manage the information of agricultural and biological engineering experts can improve the working efficiency of managers.

\section{System design}

The system adopted a top-down structured design method ${ }^{[10-12]}$. When designing, first consider the system's tasks, condition, needs, and limitations, and then design a modular architecture that could meet the system requirements as a whole, and design each subsystem or sub-module. Considering the requirements of interactivity, fast processing speed, and convenient maintenance, the system adopted $\mathrm{B} / \mathrm{S}$ architecture, the database design considers expandable data fields, and adopted component software development technology to ensure the practicability of the system, flexibility, reusability, interactivity, and scalability.

The system was designed with humanization and a friendly interface. In the user management module, the administrator role could import excel tables into the SQL Server database and implemented the function of exporting excel data from the SQL Server database. The framework of the system was Browser/Server structure, referred to as B/S structure. Unlike the $\mathrm{C} / \mathrm{S}$ structure, the client did not need to install special software, only a browser was required. The browser interacted with the database through the web server, which could easily work under different platforms. This design could make data administrators faster and easier to maintain data. The overall design architecture of this system is shown in Figure 1.

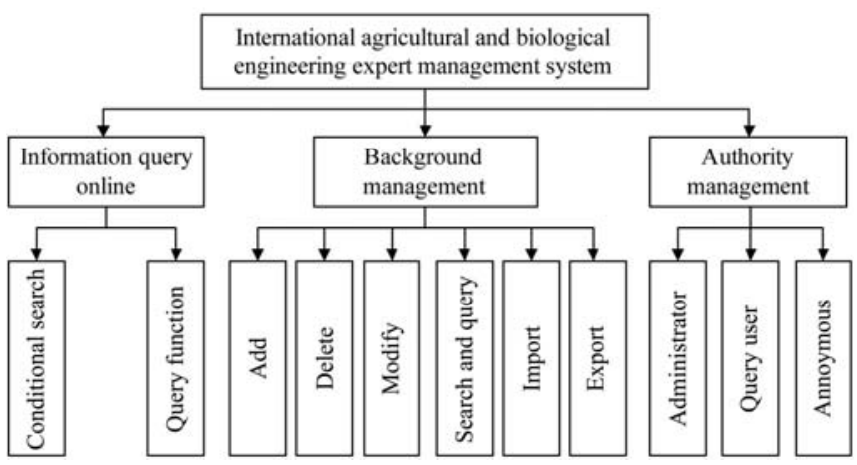

Figure 1 System design structure diagram

This software background of the system used $\mathrm{C \#}$ as the development language, and the foreground used JavaScript technology and bootstrap ${ }^{[13-16]}$. Adopted ASP.NET MVC as the web development framework, and used the Entity Framework to operate the SQL Server background database $\mathrm{e}^{[17-21]}$.

\section{Functional design and system development}

\subsection{Database design}

Information resources are an important foundational achievement of system construction and the physical basis of information sharing and exchange, including the original database and the results database. All kinds of data provide data guarantees for the business application layer through a unified database standard.

The data access layer is a component layer used to complete access to the background database and provide services for the business processing layer. The data access component of the dedicated application system uses the Entity Framework database engine to access the background database based on the B/S model to achieve the function of adding data, modifying data, deleting data, and searching queries by criteria.

The tables designed according to the requirements of this platform mainly include user table, role table, user_role table, and expert information table. In the data field design process, naming conventions and compatibility requirements need to be considered. The contents of the designed data fields are shown in Tables 1-4.

Table 1 shows the design of the data structure of users. Table 2 shows the design of the data structure with roles, including administrators, registered users and anonymous users. Table 3 is used to build the relationship between users and roles, and Table 4 shows the data structure of international agricultural and biological engineering expert data fields.

Table 1 AspNetuser

\begin{tabular}{lcc}
\hline \multicolumn{1}{c}{ Name } & Data type & Allow nulls \\
\hline$[$ Id] & NVARCHAR (128) & NOT NULL \\
{$[$ Email] } & NVARCHAR(256) & NULL \\
[EmailConfirmed] & BIT & NOT NULL \\
[PasswordHash] & NVARCHAR(MAX) & NULL \\
[SecurityStamp] & NVARCHAR(MAX) & NULL \\
{$[$ PhoneNumber] } & NVARCHAR(MAX) & NULL \\
{$[$ PhoneNumberConfirmed] } & BIT & NOT NULL \\
{$[$ TwoFactorEnabled] } & BIT & NOT NULL \\
{$[$ LockoutEndDateUtc] } & DATETIME & NULL \\
{$[$ LockoutEnabled] } & BIT & NOT NULL \\
{$[$ AccessFailedCount] } & INT & NOT NULL \\
[UserName $]$ & NVARCHAR(256) & NOT NULL \\
\hline
\end{tabular}


Table 2 Role

\begin{tabular}{|c|c|c|}
\hline Name & Data type & Allow nulls \\
\hline [Id] & NVARCHAR (128) & NOT NULL \\
\hline [Name] & NVARCHAR (256) & NOT NULL \\
\hline [Discriminator] & NVARCHAR (128) & NOT NULL \\
\hline \multicolumn{3}{|c|}{ Table 3 AspNetUser_Role } \\
\hline Name & Data Type & Allow Nulls \\
\hline [UserId] & NVARCHAR (128) & NOT NULL \\
\hline [RoleId] & NVARCHAR (128) & NOT NULL \\
\hline \multicolumn{3}{|c|}{ Table 4 Expert } \\
\hline Name & Data Type & Allow Nulls \\
\hline [Id] & NVARCHAR (128) & NOT NULL \\
\hline [Surname] & NVARCHAR(MAX) & NOT NULL \\
\hline [MiddleName] & NVARCHAR(MAX) & NULL \\
\hline [Gender] & INT & NOT NULL \\
\hline [Nationality] & NVARCHAR(MAX) & NULL \\
\hline [Language] & NVARCHAR(MAX) & NULL \\
\hline [Education] & INT & NOT NULL \\
\hline [Email] & NVARCHAR(MAX) & NOT NULL \\
\hline [TechnicalTitle] & NVARCHAR(MAX) & NULL \\
\hline [Major] & NVARCHAR(MAX) & NOT NULL \\
\hline [ResearchInterest] & NVARCHAR(MAX) & NULL \\
\hline [Phone] & NVARCHAR(MAX) & NULL \\
\hline [Organization] & NVARCHAR(MAX) & NULL \\
\hline [Address] & NVARCHAR(MAX) & NULL \\
\hline [URL] & NVARCHAR(MAX) & NULL \\
\hline [OtherMessage] & NVARCHAR(MAX) & NULL \\
\hline
\end{tabular}

This system has the function of searching according to keywords and provides functions of adding, querying, modifying, and deleting data. In addition, in order to meet the needs of users, the results can be exported to Excel reports, and corresponding import and maintenance can be performed.

\subsection{Module design}

The main functional modules implemented by the service platform are the following three functional modules: background management, data query, and authority management.

Functions of the authority management module: (1) The administrator user (Admin) role of the system initialization can disable and delete members of the information query role users (Query user) as required. The administrator user (Admin) can restore the disabled information query role users (Query user) according to actual needs, and the deleted query role (Query user) users can only be re-added. (2) The administrator role user creates information query role users who can only have the function of query or conditional query.

The background management module has the following functions: (1) The function of adding, deleting, modifying and query for expert information. (2) Import and export of expert information.

The function of the information query online module is (1) query the detailed information of agricultural and biological engineering expert information. (2) Search for expert information by keywords.

\subsubsection{Background management module}

The background management module realizes the function of data management. The design of the background management module is shown in Figure 2.

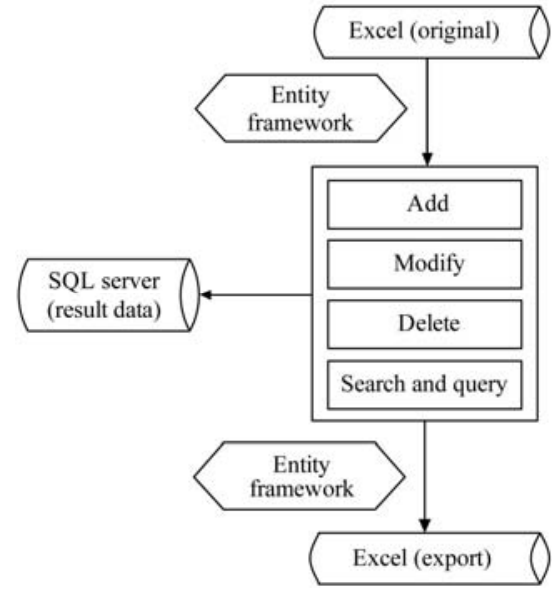

Figure 2 Background management module process structure diagram

1) Main interface of background management

After logging in the system as an administrator user, the main interface of the expert management system implements functions such as information display, search according to conditions, and data import and export. The details are shown in Figure 3.

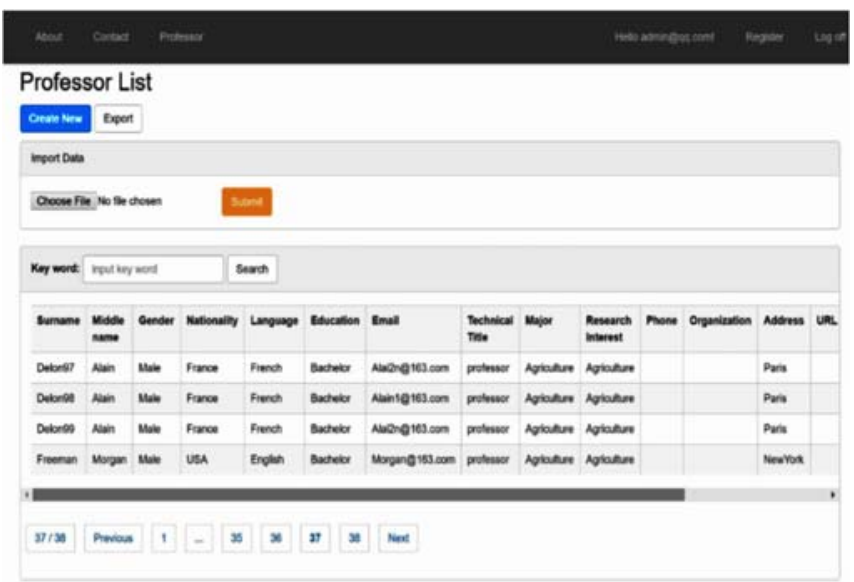

Figure 3 Main interface of background management

The data access technology uses Entity Framework technology. The Entity Framework uses the abstract data structure method to convert each database object into an application object (Entity), and the data fields are replaced with attributes, and the relationships are converted into combined attributes. Let the $\mathrm{E} / \mathrm{R}$ model of the database be completely transformed into an object model so that programmers can use the most familiar programming language to call and access. Under the abstract structure, it is a highly integrated and corresponding structure of the concept layer, the corresponding layer and the storage layer, and the data provider supporting the Entity Framework, so that the data access work can be carried out smoothly and completely.

2) Ability to modify data

After logging in as an administrator of the international agricultural and biological engineering expert management system, the data fields can be modified. The data fields of the database are including Surname, Middle name, Gender, Nationality, Language, Educational level, Email, Technical Title, Major, Research Interest, Phone, Organization, Address, URL, and another message, which is the related interface is shown in Figure $4 . \quad$ The function of adding or modifying as required is implemented. When you click Save, a corresponding dialog box can pop up to prompt the user whether to modify the original record. This record reminds the user whether to modify the original record. 
If there is no such record in the database, it is saved directly.

\section{About Contact Professor}

\section{Edit}

\section{Professor}

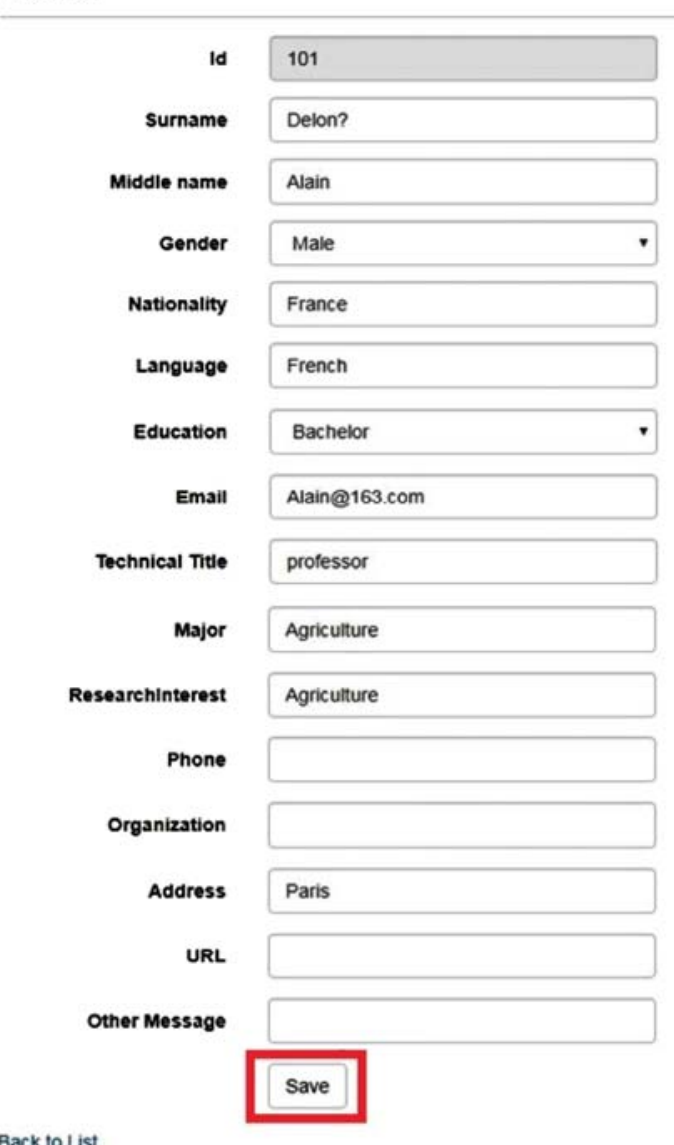

Back to List

Figure 4 Edit and modify function

3) Function of deleting data

Administrator Users can query related data. If they need to be deleted, the user can click the delete button to delete the data. After clicking "Delete", the user can enter the expert information delete confirmation function of the international agricultural and biological engineering expert management system.

4) Search and query function by condition

The system implements a search function. By entering keywords in the relevant interface, you can query international agricultural and biological engineering experts according to the keywords online. The system can search for information when using the keywords "Technical Title”, “Major”, and "Surname”, etc. The details are shown in Figure 5. For example, Search with Major as the keyword, enter "Agriculture" in the Keyword dialog box and click the "Search Button", then search for related information for international agricultural and biological engineering experts. The system implements cross-language searches and matches based on the language type and content of keywords.

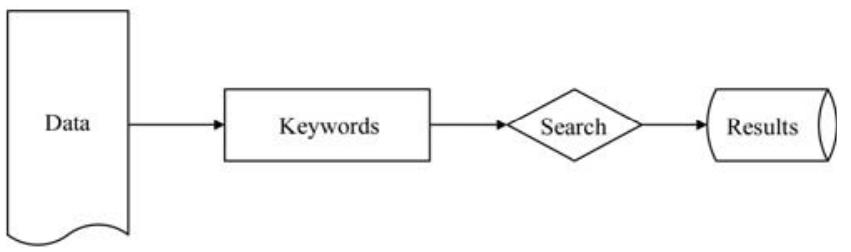

Figure 5 Conditional search flow chart
The specific implementation code is as follows:

$$
\text { // GET: Professors }
$$

[Authorize(Roles = "Queryusers")] public ActionResult Index(string keyword = “”, int pageindex = 0) \{

List $<$ Professor $>$ list;

keyword = keyword.Trim();

int count;

if (keyword != string.Empty)

\{

list $=\mathrm{db}$.Professors

.Where $(\mathrm{p}=>$

p.MiddleName.Contains(keyword) \|

p.Surname.Contains(keyword) ॥

p.Major.Contains(keyword) |

p.TechnicalTitle.Contains(keyword)

)

.OrderBy(p $=>$ p.Surname $)$

.Skip $(($ pageindex $) * 4)$

Take(4)

.ToList();

count $=$ db.Professors.

Count $(\mathrm{p}=>$

p.MiddleName.Contains(keyword) ॥

p.Surname.Contains(keyword) \|

p.Major.Contains(keyword) |

p.TechnicalTitle.Contains(keyword));

\}

else

\{

list $=\mathrm{db}$.Professors

.OrderBy $(\mathrm{p}=>$ p.Surname $)$

.Skip ((pageindex) * 4)

.Take(4).ToList();

count $=\mathrm{db}$.Professors.Count();

\}

this.ViewBag.pageIndex = pageindex;

this.ViewBag.pagecount $=$ count $/ 4+1$;

this.ViewBag.keyword = keyword; return View(list);

5) Data import and export function

After logging in with an administrator account, the system implements the function of importing information from international agricultural and biological engineering experts. Users can click the "Choose File" button to import the external data into the SQL Server database, and they can get the international agricultural and biological engineering experts excel sheet through the export function.

6) Password modification function

The administrator role can modify the password to ensure the security of agricultural and biological engineering expert information.

\subsubsection{Information query function module}

In this module, the information search function online was realized. Information searchers can search and query agricultural and biological engineering expert information as needed. In addition, the information query user can also modify the login password.

4.2.3 User background management module

The system runs for the first time and automatically creates the 
user “admin@qq.com”, sets the account user name, email, password, and distribute the admin role.

Different roles have different permissions. For example, the administrator account and the information query account have different authorities. The international agricultural and biological engineering expert management system assigns different authorities and functions to different types of users.

The administrator can create the information query role users, and the administrator user (Admin) can disable and delete the information query role users (Query user) as needed. The administrator user (Admin) can restore the disabled information query role users (Query user) according to actual needs, and the deleted query role users (Query user) can only be added again.

The administrator role (Admin) can add, delete and modify, and query related information of international agricultural and biological engineering experts.

The role of information query role users (Query user) cannot modify, delete, or add relevant information of international agricultural and biological engineering experts, and can only query information of international agricultural and biological engineering experts.

Guest users cannot query information about experts. Due to the privacy protection of personal information of international agricultural and biological engineering experts, guest users cannot query the expert's information. The functions of different roles are listed in Table 5.

Table 5 Function of different users

\begin{tabular}{|c|c|c|}
\hline No. & User & Function \\
\hline 1 & Administrator & $\begin{array}{l}\text { adding, deleting and modifying, and querying experts } \\
\text { related information }\end{array}$ \\
\hline 2 & Query user & query experts related information \\
\hline 3 & anonymous & none \\
\hline
\end{tabular}

\subsection{System development}

According to the above design principles, C\# was used as the development language under the windows platform and the JavaScript technology and bootstrap style were used in the foreground. ASP.NET MVC was adopted as the web development framework, and the Entity Framework was used to operate the SQL Server background database. The system has the function of searching and querying agricultural and biological engineering expert information according to keywords and has realized the functions of adding, deleting, modifying data records, and can generate spreadsheets and import spreadsheet data through the platform.

In the conditional search module, this module provides a conditional querying function, and users can get the agricultural and biological engineering expert information with the keywords of Technical Title, Major, Surname, and Middle name. The user can export the query data to an external file as required and can import external data into the database. The platform provides more accurate data services to the managers of agricultural and biological engineering databases, which can provide the think tank resources to help the agricultural and biological engineering discipline rapid development.

This study used the BS framework, which is different from the CS framework. C/S structure, that is, Client/Server structure, is a type of well-known software system architecture. By reasonably distributing tasks to Client and Server, the communication overhead of the system is reduced and can be fully utilized advantages of the hardware environment of both ends. Early software systems used this as the preferred design standard. The $\mathrm{B} / \mathrm{S}$ structure, namely Browser/Server structure, is a change or improved structure to the C/S structure with the rise of internet technology. Under this structure, the user interface is completely implemented through the WWW browser, and part of the transaction logic is implemented on the front end, but the main transaction logic is implemented on the server-side, forming a so-called 3-tier structure.

$\mathrm{C} / \mathrm{S}$ has a fast response speed and is generally used in a local area network, but the development and maintenance costs are high; $\mathrm{B} / \mathrm{S}$ can achieve cross-platform, zero client maintenance, but the personalization ability is low and the response speed is slow. Compared with the BS framework, the C/S framework has different environmental requirements. $\mathrm{C} / \mathrm{S}$ users are fixed and generally only used in local area networks. They require the same operating system. If different operating systems need to be developed with different versions, the requirements for computer configuration are also high. B/S requires an operating system and a browser. It has nothing to do with the operating system platform and has lower requirements for the client computer configuration.

\section{Conclusions}

This study developed the international agricultural and biological engineering expert management system, and the platform provides management for the maintenance of expert information on agriculture and biological engineering. This study can help the agricultural and biological engineering discipline rapid development and give us a tool to acquire related information to serve the field.

This study used C\# as the development language, used JavaScript technology, Entity Framework database technology, etc., focused on the existing agricultural and biological engineering expert data, and built a safe, reliable, practical, and scalable agricultural and biological engineering platform based on Web mode. The expert information service platform can meet the actual needs of agricultural and biological engineering expert information management. The platform's design focused on simplicity, complete functions, easy to use, flexible and friendly user interface to facilitate user management and use.

The promotion and use of the developed platform can help improve the efficiency of data maintenance for managers in agriculture and biological engineering, which can reduce the cost of data maintenance, and have a positive role in accelerating the development of disciplines in the field of agriculture and biological engineering.

\section{Acknowledgements}

This work was financially supported by the China Science and Technology Association Innovation-Driven Engineering Demonstration Project (Grant No. ZLGC201901-12).

\section{[References]}

[1] Batlang U, Tsurupe G, Segwagwe A, Obopile M. Development and application of modern agricultural biotechnology in Botswana: The potentials, opportunities and challenges. GM Crops \& Food, 2014; 5(3): 183-194.

[2] da Silva Dias J C. Plant breeding for harmony between modern agriculture production and the environment. Agricultural Sciences, 2015; 6(1): 87-116.

[3] Li Y. Japan's modern agriculture and its effects on agricultural development of Anhui Province. Asian Agricultural Research, 2017; 5: 52-55. 
[4] Hernando M G, Williams K. Examining the link between funding and intellectual interventions across universities and think tanks: a theoretical framework. International Journal of Politics Culture \& Society, 2018; (2): $1-14$.

[5] Zhao C. State-of-the-art and recommended developmental strategic objectives of smart agriculture. Smart Agriculture, 2019; 1(1): 1-7. (in Chinese)

[6] Köllner P, Zhu X F, Abb P. Understanding the development of think tanks in mainland China, Taiwan, and Japan. Pacific Affairs, 2018; 91(1): 5-26.

[7] Mcafee A, Brynjolfsson E. Big Data: The management revolution. Harvard Business Review, 2012; 90(10): 60-66, 68, 128.

[8] Hu B, Deng C, Ye J. Design and implementation of visual electronic commerce based on browser/server architecture. Advanced Materials Research, 2011; 1290: 336-339.

[9] Li W, Wang W, Zhao Y. Research and development on survey and statistical analysis software of resident travel od based on B/S mode. Procedia Social and Behavioral Sciences, 2013; 96: 1453-1459.

[10] Du C, He Y. An online ticket management platform for scenic spots based on B/S mode. In: 2014 fifth international conference on intelligent systems design and engineering applications, Hunan: IEEE, 2014; pp.186-190.

[11] Köpke J, Eder J, Künstner M. Top-down design of collaborating processes. In: The 16th International Conference, 2014; pp.336-345.

[12] Tam W C, Busenberg S N. Practical experience in top-down structured software production in an academic setting. Acm Sigcse Bulletin, 1977;
9(1): 31-36.

[13] Shen J B, Lei Y C, Wang G M, Fu L Y. Research on technology of forestry data integration for county-level. Computer Application and Software, 2018; 35(6): 64-69. (in Chinese)

[14] Skeet J. C\# in depth, Second Edition. Manning, 2010; 554p.

[15] Steve F. Pro typescript: Application-scale JavaScript development. Berkeley: Apress, 2018; 287p.

[16] Na Y, Kim S W, Han Y. Javascript parallelizing compiler for exploiting parallelism from data-parallel html5 applications. Acm Transactions on Architecture \& Code Optimization, 2016; 12(4): 64. doi: 10.1145/ 2846098.

[17] Sandnes F E, Eika E. A simple MVC-Framework for local management of online course material. In: Uskov V, Howlett R, Jain L (Ed.). International Conference on Smart Education and Smart E-Learning, Cham: Springer, 2017; 75: 143-153.

[18] Sandnes F E, Eika E. A simple MVC-Framework for local management of online course material. In: Uskov V, Howlett R, Jain L (Ed.). International Conference on Smart Education and Smart E-Learning, Cham: Springer, 2017; 75: 143-153.

[19] Mackey A. Introducing. NET 4.0. Berkeley: Apress, 2010; 504p.

[20] Tasoulas E, Varras G, Tsirogiannis I, Myriounis C. Development of a GIS application for urban forestry management planning. Procedia Technology, 2013; 8: 70-80.

[21] Jorgensen A, Wort S, LoForte R, LeBlanc P, Knight B. Professional Microsoft SQL Server 2012 administration, Wiley \& Sons, 2012; 30(4): $667-675$. 